\title{
MANEJO TRADICIONAL DE PLANTAS EM REGIÕES NEOTROPICAIS
}

\author{
Ulysses Paulino de Albuquerque ${ }^{1}$
}

Recebido em 01/07/1999. Aceito em 13/10/1999

\begin{abstract}
RESUMO - (Manejo tradicional de plantas em regiões neotropicais). É apresentada breve revisão sobre os principais aspectos do manejo tradicional de plantas exercidos nas regiões neotropicais, tendo como objetivos: 1 . descrever como algumas populações tradicionais manejam seus recursos vegetais; 2 . identificar as principais estratégias empregadas; 3 . ressaltar a contribuição desse conhecimento para o desenvolvimento sustentável e manejo de recursos vegetais. São caracterizados e discutidos dois tipos de manipulação dos recursos vegetais: o de comunidade e o de espécies individuais. É possível verificar que os sistemas de conhecimento tradicional podem contribuir para o desenvolvimento sustentável, visto que algumas populações locais manejam as plantas com base em concepção sistêmica do ambiente e atitude de profunda reverência para com a natureza.
\end{abstract}

Palavras-chave - florestas tropicais, manejo tradicional, agroecossistemas, Etnobotânica

\begin{abstract}
Traditional management of plants in the Neotropical region). The main aspects of traditional plant management techniques exercised in the Neotropic are briefly reviewed. The aims of this work are: 1 . to describe how some traditional populations, especially those located in Neotropical areas, manage natural resources; 2 . to identify the main strategies employed; 3 . to emphasize the contribution of traditional techniques to the sustainable development and management of natural resources. Two types of resource management are characterized and discussed: community management, and individual species management. In view of the fact that some traditional populations manage natural resources based on an integrated conception of nature, it is possible to verify that traditional knowledge systems can contribute to sustainable development.
\end{abstract}

Key words - tropical forests, traditional management, agroecosystems, Ethnobotany

\section{Introdução}

Muito se tem discutido sobre o impacto negativo da sociedade humana sobre a biodiversidade, em especial pelo forte desequilíbrio gerado pelas sociedades industrializadas modernas que têm provocado, entre outras coisas, a redução da diversidade genética e a superexploração e extinção de muitas espécies animais e vegetais (Caballero 1994). Todavia, pouco se tem estudado sobre como populações tradicionais exploram, conservam e enriquecem a biodiversidade (Caballero 1994), e influenciam a distribuição de plantas que lhes são úteis, como relataram Albuquerque \& Andrade (1998) com relação ao gênero Ocimum L. (Lamiaceae).

A acumulação de informações sobre uso de recursos naturais por populações tradicionais tem oferecido aos cientistas modelos de uso sustentável desses mesmos recursos (Albuquerque 1997, 1999). Pode-se dizer que um dos argumentos básicos defendidos é que essas populações sabem

1 Departamento de Botânica, Laboratório de Etnobotânica e Botânica Aplicada (LEBA), CCB, Universidade Federal de Pernambuco, CEP 50670-901, Recife, PE, Brasil. e-mail: upa@npd.ufpe.br 
usar e conservar seus recursos biológicos (Posey 1990). Em função disso, estudos dentro dessa perspectiva não podem estar ausentes das discussões sobre meio ambiente.

Com base nesse pressuposto, Szabó (1997) apresentou o conceito de etnobiodiversidade como o estudo da diversidade biológica influenciada não apenas pelas condições ecológicas mas também pelas tradições culturais e a experiência ecológica acumulada por comunidades humanas mais ou menos tradicionais durante o manejo sustentado de seu ambiente. Muitos pesquisadores já têm demonstrado tais afirmativas, desenvolvendo ou adaptando métodos para testar hipóteses relacionadas ao manejo tradicional de plantas (Caballero 1994; Mapes et al. 1996, 1997; Casas \& Caballero 1996; Casas et al. 1997).

Através da reunião de dados dispersos na literatura sobre o manejo tradicional dos recursos naturais e a sua apropriação por populações tradicionais nas regiões neotropicais, serão sumariados alguns dos seus principais aspectos e implicações, sem pretender extensa revisão.

\section{Métodos de manejo tradicional}

Dados referentes ao manejo e à apropriação dos recursos naturais nas regiões neotropicais foram compilados da literatura (Balée 1989; Alcorn 1989; Balée \& Gély 1989; Anderson \& Posey 1989; Hecht \& Posey 1989; Johnson 1989; Irvine 1989; Medellín-Morales 1990). Através de sua análise, procurou-se identificar um modelo de manejo comum aos grupos que já foram esıudados, nas regiões neotropicais, refletindo padrões de técnicas e práticas. Nessa perspectiva analítica, o manejo dos recursos nas florestas tropicais obedece a dois tipos de manipulação: a de comunidades e a de espécies individuais. Esse enquadramento foi assim reconhecido por outros pesquisadores: manipulação de vegetação em massa e manipulação de plantas individuais (Alcorn 1981); manipulação em massa (manejo silvícola) e como indivíduos (manejo arborícola) (Medellín-Morales 1990); manejo sucessional (Irvine 1989) que inclui os dois métodos acima citados (reconhecidos neste texto) e foi definido como a alteração do curso natural da sucessão nas florestas tropicais pelo favorecimento da distribuição de espécies úteis. Para ilustrar o manejo em massa, tomar-se-á como exemplo o bem documentado e estudado caso dos índios Kayapó do Brasil Central (Kerr \& Posey 1984; Anderson \& Posey 1989; Balée 1989; Balée \& Gély 1989; Posey 1984,1987a,b; Hecht \& Posey 1990). De modo geral, os estudos focalizando o manejo tradicional das florestas tropicais foram realizados com grupos indígenas da Amazônia.

As informações e exemplos sobre o manejo de espécies individuais foram baseados nas pesquisas desenvolvidas principalmente com plantas comestíveis no México, considerando que a Mesoamérica é um dos centros mais importantes de origem da agricultura e domesticação de plantas do mundo, resultado de uma longa interação entre populações humanas e plantas (Caballero 1990; Salinas et al. 1993). Ambas as formas de manipulação afetam a estrutura e a composição das comunidades e influenciam a evolução de espécies individuais. As formas de manipulação antropogênica dos recursos vegetais acrescentaram importante diversidade genética à já existente por causas naturais, visto que o homem vai selecionando e mantendo diferentes genótipos que lhe são úteis (Caballero 1990).

\section{Manipulação de comunidades}

Muitas pesquisas têm revelado que as culturas indígenas das regiões tropicais manipulam com ótimo aproveitamento os seus recursos naturais. Balée (1989) estima que aproximadamente $11,8 \%$ da floresta de terra firme da Amazônia é antropogênica, sendo que a manipulação humana desse recurso foi fator cultural de grande importância na adaptação do homem à Amazônia. A existência de extensas áreas de zonas antropogênicas nas florestas tropicais deve-se ao sistema de manejo de plantas exercido pelas diversas etnias. A Tab. 1 sintetiza os diferentes métodos gerais de manejo tradicional reportados na literatura. A Tab. 2, por sua vez, evidencia a diversidade dessas práticas tradicionais quando inseridas em um sistema complexo de manejo dos recursos naturais e uso da terra. Nela é possível observar os diferentes métodos de manejo 
Tabela 1. Síntese dos métodos de manejo realizados por comunidades tradicionais nas regiões neotropicais. Referências: a= Alcorn 1981, b= Alcorn 1989, c= Balée \& Gély 1989, d= Irvine 1989, e= Posey 1987a, f= Medellín-Morales 1990, g= Caballero 1994, h= Casas \& Caballero 1996, i= Casas et al. $1997, \mathrm{j}=$ Mapes et al. 1996, l= Mapes et al .1997.

\begin{tabular}{|c|c|}
\hline Manejo de comunidades & Manejo de espécies individuais \\
\hline $\begin{array}{l}\text { 1. Plantações de espécies. a.b.c.d.e } \\
\text { A. Plantas domesticadas. } \\
\text { B. Plantas semi-domesticadas. } \\
\text { 2. Transplante de espécies úteis (floresta primária } \\
\text { ou secundária).e }\end{array}$ & $\begin{array}{l}\text { 1. Plantas coletadas. }{ }^{\text {ghti }} \\
\text { 2. Plantas semi-domesticadas (sob manejo incipiente). }{ }^{\text {ghi,j.1 }} \\
\text { A. Tolerância. } \\
\text { B. Promoção. } \\
\text { C. Proteção }\end{array}$ \\
\hline $\begin{array}{l}\text { 3. Capinação seletiva. } \\
\text { 4. Poda do dossel. }\end{array}$ & 3. Plantas cultivadas \\
\hline
\end{tabular}

Tabela 2. Alguns exemplos de sistemas de manejo de solo, vegetação, água etc., usados por camponeses na América Latina. Traduzido e reproduzido de Altieri (1988).

\begin{tabular}{|c|c|c|}
\hline Limitação ambiental & Objetivo & Práticas de manejo \\
\hline Espaço limitado & $\begin{array}{l}\text { Maximizar o uso de recursos } \\
\text { ambientais e terra disponível. }\end{array}$ & $\begin{array}{l}\text { Policulturas, agroflorestas, hortos familiares, } \\
\text { zonificação altitudinal, fragmentação da propriedade, } \\
\text { rodízios. }\end{array}$ \\
\hline Terrenos em declive & $\begin{array}{l}\text { Controlar a erosão, conservar } \\
\text { a água. }\end{array}$ & $\begin{array}{l}\text { Terraços, barreiras vivas e mortas, "mulching", } \\
\text { coberturas vivas contínuas, alqueive. }\end{array}$ \\
\hline Fertilidade marginal do solo & $\begin{array}{l}\text { Manter a fertilidade e reciclar } \\
\text { a matéria orgânica. }\end{array}$ & $\begin{array}{l}\text { Alqueives naturais ou melhorados, rodízios e/ou } \\
\text { associações com leguminosas, "composting", adubos } \\
\text { verdes e orgânicos, pastoreio em campos e em } \\
\text { alqueives ou depois da colheita o uso de sedimentos } \\
\text { aluviais etc. }\end{array}$ \\
\hline Inundações ou excessos de água & $\begin{array}{l}\text { Integrar a agricultura e as } \\
\text { massas de água. }\end{array}$ & Cultivos em campos elevados. \\
\hline $\begin{array}{l}\text { Chuvas escassas ou pouco } \\
\text { predizíveis }\end{array}$ & $\begin{array}{l}\text { Conservar a água e otimizar o } \\
\text { uso da umidade disponível. }\end{array}$ & $\begin{array}{l}\text { Uso de cultivos tolerantes a seca, "mulching", } \\
\text { policulturas, uso de ciclo curto etc. }\end{array}$ \\
\hline $\begin{array}{l}\text { Extremos de radiação e/ou } \\
\text { temperatura }\end{array}$ & Melhorar o microclima. & $\begin{array}{l}\text { Redução ou incremento da sombra, podas, } \\
\text { espaçamento de cultivos, uso de cultivos que toleram } \\
\text { sombra, manejo de vento com quebra-vento, cercas } \\
\text { vivas, trabalho mínimo, policultivos, agroflorestas etc. }\end{array}$ \\
\hline Incidência de pragas & $\begin{array}{l}\text { Proteger os cultivos, reduzir as } \\
\text { populações de pragas. }\end{array}$ & $\begin{array}{l}\text { Supersemeadura, tolerância de certos danos, uso de } \\
\text { variedades resistentes, semeadura em épocas de baixo } \\
\text { potencial de pragas, manejo do habitat para } \\
\text { incrementar inimigos naturais, uso de plantas } \\
\text { repelentes etc. }\end{array}$ \\
\hline
\end{tabular}

exercidos por camponeses na América Latina e sua relação com as limitações ambientais a que estão expostos. Esses métodos correspondem a um modelo de manejo praticamente adotado em todas as populações, indígenas ou não, que exercem o hábito da agricultura itinerante. O manejo de comunidades ou de espécies individuais não são mutuamente excludentes no tempo e no espaço, e os dois métodos podem ser exercidos concomitantemente (Irvine 1989). A diferença entre os métodos é que, no primeiro, as ações estão concentradas na comunidade como um todo, e no segundo, concentram-se sobre espécies particulares.

Os processos sucessionais de manejo apontados na Tab. 1 podem ser exemplificados no caso dos índios Kayapó que, intencionalmente, manipulam vasto repertório de plantas úteis concentradas nos velhos campos de cultivo e capoeiras, para onde espécies, tanto da floresta primária, quanto secundária foram transplantadas. 
O hábito de transplantar espécies para locais junto a trilhas e acampamentos produziu artificialmente campos na floresta que reproduzem as "ilhas naturais de recursos", locais que concentram plantas e animais úteis. Mais ainda, a agricultura itinerante Kayapó mantém em um mesmo campo diferentes espécies, com hábitos e exigências nutricionais também distintos, possibilitando, dessa forma, menor impacto da chuva sobre o solo, e menor competitividade entre as espécies por nutrientes. Essa solução reduz o impacto das atividades agriculturais humanas sobre o ambiente. Outra observação interessante sobre o manejo dos Kayapó é a criação dos chamados "apête" (ilhas de floresta em savana). O estudo de Anderson \& Posey (1989) revelou que, do total das espécies contabilizadas nessas ilhas, $98 \%$ delas eram úteis para os Kayapó e que grande percentagem dessas plantas $(75 \%)$ haviam sido plantadas pelos índios. Os autores alertaram para o fato de que, embora à primeira vista essas ilhas pareçam naturais, são basicamente antropogênicas, comprovando que a atividade Kayapó exerce forte impacto na estrutura e composição da savana.

Os sistemas tradicionais, de modo geral, envolvem o corte da vegetação e a queimada de árvores com vistas à formação dos campos para cultivo de plantas diversas. Essa técnica requer apurado conhecimento dos tipos de solo e um sistema próprio de classificação das florestas (Carneiro 1987; Hecht \& Posey 1989; Ribeiro 1990). Guardadas as devidas proporções e realizadas segundo o saber indígena, essas técnicas possibilitam o rejuvenescimento da floresta (Taylor 1997). O mesmo autor também acrescenta ao caso Kayapó as informações que colheu junto aos Ianomami: Os Ianomami e os Caiapó vivem na floresta e são parte dela. Se eles a destruírem, destruirão a si próprios. Portanto, eles fazem campos de proporções modestas e plantações suficientes para suas necessidades. São os agricultores (ou investidores) não-índios que ordenam a destruição da floresta, que provavelmente nunca viram, a fim de instalar plantações ou criações de gado totalmente impróprias. Isso, é claro, tem que ser feito em grande escala, a fim de que possam ter sua margem de lucro como desejam. As enormes clareiras resultantes ficam muito acima da capacidade de regeneração de qualquer floresta saudável.

O manejo de comunidades reflete um padrão que envolve plantação, transplante, proteção de espécies e outras ações, visando o aproveitamento total dos recursos e compatibilizando aspectos temporais e espaciais. Mediante o manejo adequado das espécies vegetais e do solo, os ecossistemas são manipulados em sistema de rotatividade, garantindo o restabelecimento dos mesmos após período de uso. O hábito de transplantar espécies, descrito por Posey (1987b) como agricultura itinerante, é uma etapa importante dos sistemas de manejo de comunidades. Os estudos de Irvine (1989) e Medellín-Morales (1990) revelam que outras práticas além das relatadas, como a capinação seletiva ("desyerbe selectivo") e a poda do dossel, afetam a sucessão vegetal nos chamados "mosaicos de vegetação antropogênica" (campos formados depois do cultivo), o que pode resultar em maior diversidade, também em espécies úteis.

\section{Manipulação de espécies individuais}

A manipulação de espécies individuais corresponde a atenção especialmente devotada para determinadas plantas de grande interesse. Dentre as três categorias de plantas apontadas na Tab. 1, de acordo com o seu nível de manipulação e pressão de seleção exercida, as semi-domesticadas (ou sob manejo incipiente) concentram determinadas ações: tolerância, que permite aos indivíduos selecionados permanecerem em determinados lugares, como por exemplo em campos de milho; promoção, que prevê ações que favorecem a distribuição e a dispersão da espécie por via vegetativa ou sexual; proteção, que consiste na proteção de determinados indivíduos eliminando, por exemplo, competidores e aumentando as possibilidades de sobrevivência dessas plantas (Caballero 1994).

Essas diferentes formas de manipulação de plantas semi-domesticadas produzem importantes efeitos biológicos (Tab. 3). Casas \& Caballero (1996) verificaram em Leucaena esculenta (Moc. et Sessé ex A.D.C.) Benth. uma divergência 
morfológica em populações submetidas a diferentes formas de manejo tradicional. A domesticação de espécies de Amaranthus obedece a dois caminhos em função da forma de uso e manejo por populações humanas, indicando que plantas selecionadas para uso como verdura divergem morfologicamente daquelas das quais se usa os grãos (Mapes et al. 1996, 1997). Bom exemplo é o de A. hypocondriacus L. em que a promoção de indivíduos pela dispersão de sementes tem como efeito biológico o aumento da biomassa foliar e do seu ciclo de vida (Caballero 1994; Mapes et al. 1996). Outro caso interessante é o do cacto Stenocereus stellatus (Pfeiffer) Riccobono, amplamente empregado no México para fins alimentícios. Grupos indígenas são capazes de distinguir e classificar variações na espécie com base nas características morfológicas dos frutos, verificando-se que plantas silvestres apresentam frutos pequenos, vermelhos e de sabor acre, com pericarpo grosso e espinhoso, diferindo das plantas por eles cultivadas, que variam em uma ou mais dessas características (Casas et al. 1997). De acordo com esses autores, S. stellatus vem sofrendo um processo de seleção artificial por populações locais.

Esses estudos têm sérias implicações na detecção de novos recursos vegetais e nas discussões sobre a diversidade e evolução vegetal. Como observa Caballero (1990): La domesticación de plantas y la generación de diversidad a través de la manipulación de la variabilidad no es un evento concluido ni si limita a las plantas cultivadas que todos conocemos.

\section{Sistemas agroflorestais nas regiões tropicais}

Os sistemas agroflorestais adotam técnicas de uso da terra amplamente disseminadas nas regiões tropicais e têm como um de seus elementos a perfeita adaptação na satisfação das necessidades locais (Maydell 1979; 1984). São reportados por diferentes pesquisadores como alternativa ao uso

Tabela 3. Formas de manipulação de plantas semi-domesticadas no México e seus efeitos biológicos. Compilado de várias fontes por Caballero (1994).

\begin{tabular}{|c|c|c|c|}
\hline Espécies & Parte utilizada & Formas de manipulação & Modificações \\
\hline Brassica campestris L. & Folhas & $\begin{array}{l}\text { Épocas diferentes daquelas do } \\
\text { ciclo de vida das formas } \\
\text { selvagens }\end{array}$ & $\begin{array}{l}\text { Fenótipos com folhas abundantes, sem } \\
\text { inflorescências e com hipocótilo mais } \\
\text { grosso. }\end{array}$ \\
\hline $\begin{array}{l}\text { Jaltomata procumbens } \\
\text { (Cav.) L. Gentry }\end{array}$ & Frutos & $\begin{array}{l}\text { Indivíduos tolerados ou } \\
\text { protegidos durante os trabalhos } \\
\text { agrícolas. }\end{array}$ & $\begin{array}{l}\text { Fenótipos com menos frutos mas de maior } \\
\text { tamanho. }\end{array}$ \\
\hline Solanum mozianum Dun. & Folhas & $\begin{array}{l}\text { Uso do arado favorece a } \\
\text { reprodução vegetativa dos } \\
\text { indivíduos que crescem nos } \\
\text { campos de milho. }\end{array}$ & Gigantismo e perda da deiscência dos frutos. \\
\hline $\begin{array}{l}\text { Amaranthus } \\
\text { hypochondriacus } \mathrm{L} \text {. }\end{array}$ & Folhas & $\begin{array}{l}\text { Seleção e dispersão de grãos } \\
\text { dos indivíduos excepcionais que } \\
\text { crescem nos campos de milho. }\end{array}$ & $\begin{array}{l}\text { Aumento da biomassa foliar, redução da } \\
\text { biomassa da inflorescência, ciclo de vida } \\
\text { mais longo. }\end{array}$ \\
\hline $\begin{array}{l}\text { Leucaena esculenta } \\
\text { (Moc. et Sessé ex A.D.C.) } \\
\text { Benth. }\end{array}$ & Frutos & $\begin{array}{l}\text { Tolerância, seleção e promoção } \\
\text { de indivíduos por ocasião do } \\
\text { arroteamento pelos trabalhos } \\
\text { agrícolas. }\end{array}$ & $\begin{array}{l}\text { Redução na quantidade de compostos } \\
\text { secundários no grão, aumento do tamanho } \\
\text { do grão e da vagem. }\end{array}$ \\
\hline Opuntia spp. & Fruto & $\begin{array}{l}\text { Transplante, tolerância e } \\
\text { desenvolvimento de indivíduos } \\
\text { excepcionais na vegetação } \\
\text { natural e nos campos de cultivo. }\end{array}$ & $\begin{array}{l}\text { Maior tamanho e peso dos frutos; maior } \\
\text { variação na cor, peso e tamanho do fruto. }\end{array}$ \\
\hline $\begin{array}{l}\text { Porophyllum ruderale } \\
\text { (Jacq.) Cass. subsp. } \\
\text { macrocephalum (DC) } \\
\text { R.R. Johnson }\end{array}$ & Folhas & $\begin{array}{l}\text { Tolerância e promoção de } \\
\text { formas adventícias; cultivo. }\end{array}$ & $\begin{array}{l}\text { Redução no tamanho dos indivíduos, } \\
\text { maior biomassa foliar e alongamento das } \\
\text { folhas. }\end{array}$ \\
\hline
\end{tabular}


da terra nas regiões tropicais e podem ser caracterizados pelo consorciamento de práticas silviculturais, agriculturais e pecuárias (Maydell 1979; Anderson et al. 1985). Nesses sistemas, as populações locais realizam diferentes formas de manejo dos recursos disponíveis, como os já apontados neste texto.

Tais sistemas podem ser praticados sob vários aspectos, sendo algumas de suas características: sustentação a longo prazo; redução de riscos pela combinação de produtos de mercado e de subsistência; dependência de fontes de conhecimento e tecnologia locais, resultantes do conhecimento das populações locais sobre o ecossistema, tornando suas práticas ecológica e economicamente sustentáveis (Anderson et al. 1985). Dessa forma, os sistemas agroflorestais, pautados na experiência e técnicas tradicionais, podem constituir-se em alternativa às técnicas de cultivo altamente destrutivas e insustentáveis atualmente empregadas nas regiões tropicais. Além disso, podem prevenir a desertificação e contribuir com a recuperação de áreas degradadas e regiões semi-áridas e promover o melhoramento de pastagens ("rangelands") pelo cultivo de árvores e arbustos produtores de forragem em savanas e estepes (Maydell 1979). O mesmo autor alerta ainda que, muito embora a contribuição de tais sistemas para a economia possa ser apenas fracamente estimada, eles garantem às populações locais as condições básicas de existência e desenvolvimento.

Os últimos anos vêm presenciando o interesse dos pesquisadores pelo estudo dos "quintais" (em inglês: homegarden, housegarden; em espanhol: huerto, solar), áreas localizadas ao redor de casas ou de pequenas propriedades caracterizadas por serem área de manejo e de uso da terra. Tais estudos têm contemplado aspectos econômicos, nutricionais, sociais e da composição florística (Rico-Gray et al. 1990; Caballero 1992; Jose \& Shanmugaratnam 1993; Rugalema et al. 1994; Dharmasena \& Wijeratne 1996), sendo que no Brasil são ainda poucos os trabalhos (Anderson et al. 1985; Saragoussi et al. 1990). Ao lado de todas essas considerações, tais sistemas são, sem dúvida, locais de experimentação e de intercâmbio de germoplasma nas comunidades em que são praticados.

Thiollay (1995) enxerga os agroecossistemas tradicionais como uma das melhores possibilidades de conservar a biodiversidade, promovendo o uso sustentável dos recursos naturais. Os sistemas de produção em pequenas comunidades tradicionais têm se mostrado sustentáveis em seus respectivos contextos (Albuquerque 1999). Práticas ou técnicas de manejo tradicional visam também contornar as

Tabela 4. Comparaçōes entre dois diferentes paradigmas de sustentabilidade. Reproduzido de Cavalcanti (1995).

\begin{tabular}{|c|c|c|}
\hline Termos de comparação & Índios & EUA \\
\hline Visão de mundo & Reverência pela natureza; humildade & $\begin{array}{l}\text { Homem senhor e possuidor da natureza; } \\
\text { arrogância }\end{array}$ \\
\hline Formação de capital & $\begin{array}{l}\text { Quase nenhuma; habilitações e } \\
\text { ferramenta toscas }\end{array}$ & $\begin{array}{l}\text { Cumulativa; necessidade de volume } \\
\text { sempre crescente de investimentos (para } \\
\text { manter taxas constantes) }\end{array}$ \\
\hline Fontes de energia & Renováveis somente & $\begin{array}{l}\text { Combustíveis fósseis (fontes não- } \\
\text { renováveis); menor proporção dos } \\
\text { renováveis }\end{array}$ \\
\hline Formas de conhecimento & $\begin{array}{l}\text { Base na experiência (transmissão oral } \\
\text { pelos antigos e pelos pajés) }\end{array}$ & $\begin{array}{l}\text { Ciência moderna (transmissão sob } \\
\text { forma escrita, bibliotecas, meios } \\
\text { eletrônicos) }\end{array}$ \\
\hline Fonte de propulsão & Recursos naturais & Progresso técnico \\
\hline Uso de matéria e energia & Frugalidade, parcimônia termodinâmica & $\begin{array}{l}\text { Forte degradação entrópica; } \\
\text { esbanjamento, desperdício }\end{array}$ \\
\hline Principais objetivos econômicos & $\begin{array}{l}\text { Satisfação das necessidades básicas; } \\
\text { bem-estar comunitário }\end{array}$ & $\begin{array}{l}\text { Crescimento econômico ilimitado; lucro } \\
\text { imediato }\end{array}$ \\
\hline Tendência a longo prazo & Altamente sustentável & Insustentável \\
\hline
\end{tabular}


limitações ambientais, mantendo a diversidade. Obviamente a visão de mundo das populações locais direciona suas práticas relativas ao ambiente (Tab. 4), desenvolvendo, como aponta Altieri (1988), sistemas de manejo adaptados para superar as limitações impostas pelo meio (Tab. 2). A Tab. 4 compara ainda dois diferentes paradigmas de estilos de vida: $o$ das comunidades tradicionais (exemplificado com o caso de algumas tribos brasileiras) e o da sociedade industrializada (representada pelo padrão de consumo de recursos dos EUA).

\section{Considerações finais}

Nesse ponto vem uma pergunta à mente: em que o conhecimento dos processos de manejo tradicional pode contribuir na conservação da biodiversidade e no tão propalado desenvolvimento sustentável? Rêgo (1999) afirma que $o$ bioextrativismo das populações tradicionais pode ser definido como a intervenção, na biota dos ecossistemas naturais, pelo homem (componente da biota), baseada na racionalidade da reprodução familiar, comunitária e sobredeterminada por seu universo cultural, fundado na simbiose prática e simbólica com a natureza. Na verdade, essa idéia de um neoextrativismo que concilia os aspectos culturais e sociais no espaço em que é produzido (ou processado) pode expandir-se além das fronteiras do ecossistema amazônico (objeto da análise de Rêgo 1999), a outros importantes ecossistemas. Begossi (1998) analisa essa possibilidade para o caso da floresta atlântica, onde seria possível conciliar o uso dos recursos pelos "caiçaras" unindo a propósitos de conservação.

Com base em Altieri (1988) e Rêgo (1999) pode-se dizer que as atividades ou programas de desenvolvimento devem incluir alguns critérios básicos, aqui resumidos em apenas dois: 1 . fundamento em tecnologias fáceis e facilmente comunicáveis entre os membros da comunidade, apoiadas nos sistemas e práticas de manejo tradicional; 2 . envolvimento das populações locais no seu planejamento e execução. Mesmo observando que as práticas de manejo nas regiões tropicais são muito diversificadas, elas compartilham muitas semelhanças, principalmente no que respeita a aspectos funcionais e estruturais. Assim, a análise e os textos comentados nesta revisão permitem ressaltar três importantes pontos: 1. a melhor estratégia de conservação e desenvolvimento é aquela que permite preservar a biodiversidade existente e os fatores e técnicas culturais que também são a sua origem; 2 . necessidade de pesquisas que investiguem a base genética dos processos tradicionais de domesticação e seleção de plantas; 3. necessidade de avaliação do impacto das práticas tradicionais sobre a biodiversidade local.

É chegada a hora de reconhecer o papel dos agricultores tradicionais, indígenas ou não, bem como todo o seu saber acumulado através das gerações. Considerando, então, a conservação dos recursos genéticos de produtos agrícolas, surge a necessidade de serem empreendidos esforços no desenvolvimento rural que considerem igualmente a conservação dos recursos locais e a autosuficiência com participação no mercado, tudo isso ao nível do pequeno agricultor (Altieri \& Merrick 1987, 1997). Dessa forma, parafraseando Gottlieb et al. (1996), sem que se dê a devida importância ao conhecimento de conteúdo biológico-cultural, o desenvolvimento sustentável não passará de um apelo político sem retorno social.

\section{Agradecimentos}

$\mathrm{O}$ autor agradece à Dra. Laise de Holanda Cavalcanti Andrade, Departamento de Botânica, CCB, UFPE, pela leitura do texto; ao Dr. William Sanders, pela revisão do Abstract; aos revisores anônimos, pelas importantes sugestões.

\section{Referências bibliográficas}

Albuquerque, U. P. 1997. Etnobotânica: uma aproximação teórica e epistemológica. Revista Brasileira de Farmácia 78(3): 60-64.

Albuquerque, U. P. 1999. La importancia de los estudios etnobiológicos para establecimiento de estrategias de manejo y conservación en las florestas tropicales. Biotemas 12(1): 31-47.

Albuquerque, U. P. \& Andrade, L. H. C. 1998. Etnobotánica del género Ocimum L. (Lamiaceae) en las comunidades afrobrasileñas. Anales del Jardín Botánico de Madrid 56(1): 107-118.

Alcorn, J. B. 1981. Huastec noncrop resource management: implications for prehistoric rain forest management. Human Ecology 9: 395-417. 
Alcorn, J. B. 1989. Process as resource - the traditional agricultural ideology of Bora and Huastec Resource management and its implication for research. Advances in Economic Botany 7: 63-77.

Altieri, M. A. 1988. Sistemas agroecológicos alternativos para la producción campesina. Pp. 263-276. In: Desarrollo agrícola y participación campesina. Naciones Unidas y Comisión Económica para América Latina y el Caribe, Santiago de Chile.

Altieri, M. A. \& Merrick, L. C. 1987. In situ conservation of crop genetic resources through maintenance of traditional farming system. Economic Botany 41: 86-96.

Altieri, M. A. \& Merrick, L. C. 1997. Agroecologia e conservação in situ da diversidade de plantações nativas no terceiro mundo. Pp. 159-173. In: E. O. Wilson (Ed.), Biodiversidade. Nova Fronteira, Rio de Janeiro.

Anderson, A. B.; Gely, A.; Strudwick, J.; Sobel, G. L. \& Pinto, M. G. C. 1985. Um sistema agroflorestal na várzea do estuário amazônico (Ilha das Onças, Município de Barcarena, Estado do Pará). Acta Amazonica 15(1-2): 195-224.

Anderson, A. B. \& Posey, D. A. 1989. Management of a tropical scrub Savanna by the Gorotire Kayapó of Brazil. Advances in Economic Botany 7: 159-173.

Balée, W. 1989. The culture of amazonian forests. Advances in Economic Botany 7: 63-71.

Balée, W. \& Gély, A. 1989. Managed forest sucession in Amazonia - the Ka'apor case. Advances in Economic Botany 7: 129-158.

Begossi, A. 1998. Extractive reserve in the Brazilian Amazon: an example to be followed in the Atlantic Forest? Ciência \& Cultura 50(1): 24-28.

Caballero, J. 1990. El uso de la diversidad vegetal en México: tendencias y perspectivas. Pp. 257-290. In: E. Leff (Ed.), Medio Ambiente y desarrollo en México. Centro de Investigaciones Interdisciplinarias en Humanidades, UNAM, México.

Caballero, J. 1992. Maya homegardens: past, present and future. Etnoecológica 1(1): 35-54.

Caballero, J. 1994. La dimension culturelle de la diversité végétale au Mexique. Journal d'Agriculture Traditionel et de Botanique Appliqué 36: 145-158.

Carneiro, R. L. 1987. O uso do solo e classificação da floresta (Kuikúro). Pp. 47-58. In: B. Ribeiro (Ed.), Suma etnológica brasileira - 1. Etnobiologia. Vozes/Finep, Petrópolis.

Casas, A. \& Caballero, J. 1996. Traditional management and morphological variation in Leucaena esculenta (Fabaceae: Mimosoideae) in the Mixtec region of Guerrero, México. Economic Botany 50: 167-181.

Casas, A.; Pickersgill, B.; Caballero, J. \& Valiente, A. 1997. Ethnobotany and domestication in xoconochtli: Stenocereus stellatus (Cactaceae) in the Tehuacán valley and mixteca baja, Mexico. Economic Botany 51(3): 279 292.

Cavalcanti, C. 1995. Sustentabilidade da economia: paradigmas alternativos de realização econômica. Pp. 153176. In: C. Cavalcanti (Ed.), Desenvolvimento e sociedade: estudos para uma sociedade sustentável. Cortez/Fundaj, São Paulo.

Dharmasena, K. H. \& Wijeratne, M. 1996. Analysis of nutritional contribution of homegarding. Tropenlandwirt 97(2): 149-159.

Gottlieb, O. R.; Kaplan, M. A. C. \& Borin, M. R. M. B. 1996. Biodiversidade: um enfoque químico-biológico. Editora Universidade Federal do Rio de Janeiro, Rio de Janeiro.

Hecht, S. B. \& Posey, D. A. 1989. Preliminary results on soil management techniques of the Kayapó indians. Advances in Economic Botany 7: 174-187.

Hecht, S. B. \& Posey, D. A. 1990. Indigenous soil management in the Latin America: some implication for the Amazon basin. V.2, Pp. 57-72. In: D. A. Posey \& W.L. Overal (Ed.), Ethnobiology: implications and applications Proceedings of the first Internacional Congress of Ethnobiology. Museu Paraense Emílio Goeldi, Belém.

Irvine, D. 1989. Sucession management and resource distribution in an amazonian rain forest. Advances in Economic Botany 7: 223-237.

Johnson, A. 1989. How the Machiguenga manage resources: conservation or exploitation of nature. Advances in Economic Botany 7: 213-222.

Jose, D. \& Shanmugaratnam, N. 1993. Traditional homegardens of Kerala - a sustainable human ecosystem. Agroforest Systems 24(2): 203-213.

Kerr, W. E. \& Posey, D. A. 1984. Informações adicionais sobre a agricultura dos Kayapó. Interciência 9: 392-400.

Mapes, C.; Caballero, J.; Epistia, E. \& Bye, R. A. 1996. Morphophysiological variation in some Mexican species of vegetable Amaranthus: evolutionary tendencies under domestication. Genetic Resources and Crop Evolution 43: 283-290.

Mapes, C.; Basurto, F. \& Bye, R. 1997. Ethnobotany of quintonil: knowledge, use and management of edible greens Amaranthus spp. (Amaranthaceae) in the Sierra Norte of Puebla, Mexico. Economic Botany 51(3): 293-306.

Maydell, H. J. 1979. Agroforestry - a combination of agricultural, sylvicultural and pastoral land-use. Plant Research and Development 9: 17-23.

Maydell, H. J. 1984. The role of forestry in rural development. Plant research and development 19: 52-70.

Medellín-Morales, S. 1990. Manejo agrosilvícola tradicional en una comunidad Totonaca de la costa de Veracruz, México. V.2. Pp. 11-26 In: D. A. Posey \& W. L. Overal (Ed.), Ethnobiology: implications and applications Proceedings of the first Internacional Congress of Ethnobiology. Museu Paraense Emílio Goeldi, Belém.

Posey, D. A. 1984. Os Kayapó e a natureza. Ciência Hoje 2(12): 35-41.

Posey, D. A. 1987a. Etnobiologia: teoria e prática. Pp. $15-$ 25I. In: B. Ribeiro (Ed.), Suma etnológica brasileira 1. Etnobiologia. Vozes/Finep, Petrópolis.

Posey, D. A. 1987b. Etnobiología y ciencia "folk": su importancia para la Amazonia. Hombre y Ambiente 1: 7-26.

Posey, D. A. 1990. The application of ethnobiology in the conservation of dwindling natural resources: lost knowledge or options for the survival of the planet. V.I, 
Pp. 47-60. In: D. A. Posey \& W. L. Overal (Ed.), Ethnobiology: implications and applications Proceedings of the first Internacional Congress of Ethnobiology. Museu Paraense Emílio Goeldi, Belém.

Rico-Gray, U.; Garcia-Franco, J. G.; Chenas, A.; Puen, A. \& Sima, P. 1990. Species composition, similarity and structure of mayan homegardens in Tixpeual and Tixcacaltuyul, Yucatan, Mexico. Economic Botany 44(4): 470-487.

Rêgo, J. F. 1999. Amazônia: do extrativismo ao neoextrativismo. Ciência Hoje 25(147): 62-65.

Ribeiro, B. 1990. Classificação dos solos e horticultura Desâna. V.2, Pp. 27-50. In: D. A. Posey \& W. L. Overal (Ed.), Ethnobiology: implications and applications Proceedings of the first Internacional Congress of Ethnobiology. Museu Paraense Emílio Goeldi, Belém.

Rugalema, G. H.; Johnsen, F. H. \& Rugambisa, J. 1994. The homegarden agroforest system to Bukoba district, NorthWestern Tanzania.2. Constraints to farm productivity. Agroforest Systems 26(3): 205-214.

Salinas, J. L. V.; Fernández, A. C. \& Caballero, J. 1993. Las plantas y la alimentación entre los mixtecos de Guerrero.
Pp. 625-670. In: E. Leff. \& J. Carabias (Ed.), Cultura y manejo sustentable de los recursos naturales. Centro de Investigaciones Interdisciplinares en Humanidades, UNAM, México.

Saragoussi, M.; Martez, J. H. I. \& Ribeiro, G. A. 1990. Comparação na composição de quintais de três localidades de terra firme do Estado do Amazonas. V.1. Pp. 295-303. In: D. A. Posey \& W. L. Overal (Ed.), Ethnobiology: implications and applications - Proceedings of the first Internacional Congress of Ethnobiology. Museu Paraense Emílio Goeldi, Belém.

Szabó, V. T. 1997. The history of a concept, its possibilities and limitations. P. 71. In: Resumos do II Congresso Internacional de Etnobotánica. Mérida, Yucatán.

Taylor, K. 1997. Desmatamento e índios na Amazônia brasileira. Pp. 177-185. In: E. O. Wilson (Ed.), Biodiversidade. Nova Fronteira, Rio de Janeiro.

Thiollay, J-M. 1995. The role of traditional agroforests in the conservation of rain forests bird diversity in Sumatra. Conservation Biology 9(2): 335-353. 